\title{
Electronic shell effects and the stability of alkali nanowires
}

\author{
D.F. Urban ${ }^{\mathrm{a}}$, J. Bürki ${ }^{\mathrm{a}, \mathrm{b}}$, A.I. Yanson ${ }^{\mathrm{c}}$, I.K. Yanson ${ }^{\mathrm{d}}$, C.A. Stafford ${ }^{\mathrm{b}}$, \\ J.M. van Ruitenbeek ${ }^{\mathrm{e}}$, Hermann Grabert ${ }^{\mathrm{a}, *}$ \\ ${ }^{a}$ Physikalisches Institut, Albert-Ludwigs-Universität, Hermann-Herder-Strasse 3, D-79104 Freiburg, Germany \\ ${ }^{\mathrm{b}}$ Department of Physics, University of Arizona, Tucson, AZ 85721, USA \\ ${ }^{\mathrm{c}}$ Department of Physics, 510 Clark Hall, Cornell University, Ithaca, NY 14853, USA \\ ${ }^{\mathrm{d}}$ B. Verkin Institute for Low Temperature Physics and Enginneering, National Academy of Sciences, 310164 Kharkiv, Ukraine \\ ${ }^{\mathrm{e}}$ Universiteit Leiden, P.O. Box 9504, NL-2300 RA Leiden, The Netherlands
}

Received 9 March 2004; accepted by the guest editors

Available online 15 June 2004

\begin{abstract}
Experimental conductance histograms for Na nanowires are analyzed in detail and compared to recent theoretical results on the stability of cylindrical and elliptical nanowires, using the free-electron model. We find a one-to-one correspondence between the peaks in the histograms and the most stable nanowire geometries, indicating that several of the commonly observed nanowires have elliptical cross sections.
\end{abstract}

(C) 2004 Elsevier Ltd. All rights reserved.

PACS: 73.21.Hb; 68.65.La

Keywords: A. Nanostructures; A. Metals; D. Electronic transport

\section{Introduction}

In the past eight years, experimental research on metal nanowires has burgeoned, together with theoretical work describing these nanoscale systems in which quantum effects play an important role (see Ref. [1] for a recent review). Metal nanowires exhibit a number of interesting properties: their electrical conductance is carried by a welldefined number of quantum channels [2-5], their shot-noise is suppressed by the Pauli principle [6], and they are remarkably strong and stable [7]. The attention to this research field is accentuated by the ongoing miniaturization in the microelectronic industry and the need to understand what modified properties may be met upon further size reduction.

Crucial for answering the question of future applications is knowledge about the stability of nanowires. An important

\footnotetext{
* Corresponding author. Tel.: +49-761-203-5882; fax: +49-761203-5881.

E-mail address: grabert@physik.uni-freiburg.de (H. Grabert).
}

contribution to the energetics of metal nanowires comes from the subband structure of the conduction electrons [8-10]. Indeed, a linear stability analysis [11-14] shows that electronic shell effects can stabilize and therefore favor certain geometries. The effects of shell filling on the abundance spectra of metal clusters have been known for years. They explain the existence of clusters with 'magic numbers', corresponding to full electronic shells, which are observed more frequently than others [15]. More recently, electronic shell and supershell structures have been reported for alkali metal nanowires [16-18]. Theoretically, these effects can be understood within a stability analysis including wires with cylindrical and elliptical cross sections [14].

This paper is organized as follows: An overview on the theoretical framework is presented in the next section, including the discussion of the nanoscale free-electron model. Then experiments measuring conductance histograms of alkali metal nanowires are presented, followed by a more detailed analysis of the experimental data. It is shown that theory allows prediction of the shapes of wires 
produced in experiments. A short summary is given in Section 4.

\section{Theory}

One possibility to describe nanoscale contacts is via molecular dynamics simulations [19-21] which use shortranged interatomic potentials suitable to describe the bulk properties of metals. However, such an approach appears problematic when applied to metal nanowires, in which electron-shell effects $[16,17]$ due to transverse confinement are likely to be important. An alternative approach, motivated by the success of the jellium approximation in explaining the energetics of ultrasmall metal clusters [15, $22]$, is a nanoscale generalization of the free-electron model, developed by Stafford et al. [8]. This approach allows for analytical calculations and was successfully used in recent years to understand many properties of metal nanowires.

\subsection{Nanoscale free-electron model}

The free-electron model of a nanowire [8] treats the electrons as a non-interacting Fermi gas kept within the wire by a confining potential, which is approximated in terms of hard-wall boundary conditions. The discrete ions are replaced by a constant positive jellium background charge. This model requires good charge screening in the metal and an almost spherical Fermi-surface. It is especially suitable for alkali metals, but can also be applied to other monovalent metals such as gold.

A nanowire connecting two macroscopic electrodes is an open quantum system, for which the Schrödinger equation is most naturally formulated as a scattering problem. The fundamental quantity describing the properties of the system is the scattering matrix $S(E)$ connecting incoming and outgoing asymptotic states of conduction electrons in the electrodes. Transport properties can be expressed in terms of the submatrix $S_{12}$ describing transmission through the wire. For example, the electrical conductance $G$ is given by the Landauer formula [23]

$G=\frac{2 e^{2}}{h} \int \mathrm{d} E \frac{-\partial f(E)}{\partial E} \operatorname{Tr}\left\{S_{12}^{\dagger}(E) S_{12}(E)\right\}$

where $f(E)$ is the Fermi-Dirac distribution function.

Similarly, thermodynamic properties can be expressed in terms of the scattering matrix through the electronic density of states (DOS) $D(E)$

$D(E)=\frac{1}{2 \pi \mathrm{i}} \operatorname{Tr}\left\{S^{\dagger}(E) \frac{\partial S}{\partial E}-\frac{\partial S^{\dagger}}{\partial E} S(E)\right\}$

from which the relevant thermodynamic potential for an open system, namely the grand canonical potential $\Omega$, is obtained as

$\Omega=-k_{\mathrm{B}} T \int \mathrm{d} E D(E) \ln \left[1+\mathrm{e}^{-\frac{(E-\mu)}{k_{\mathrm{B}} T}}\right]$

where $k_{\mathrm{B}}$ is the Boltzmann constant, $T$ is the temperature and $\mu$ is the chemical potential specified by the macroscopic electrodes. Eqs. (1)-(3) include a factor of 2 for spin degeneracy.

Many fundamental phenomena of metal nanowires have been understood in terms of this nanoscale free-electron model. In particular, theoretical results on conductance quantization [24-26], cohesion $[8,26]$ and current noise [27] are in good agreement with experiments. At the meanfield level, electron-electron interactions can also be included in the model in a straightforward way [26,28,29].

In this approach, an atomic-scale contact between two macroscopic electrodes can be considered as a waveguide for conduction electrons (which are responsible for both electrical conduction and cohesion in simple metals) [8]. Each quantized mode transmitted through the contact contributes one quantum $G_{0}=2 e^{2} / h$ to its conductance, and acts as a chemical bond, delocalized through the contact, thus contributing to the cohesion of the wire.

\subsection{Stability and shell structure}

Metal nanowires are found to be remarkably stable, although one might expect them to break up because of surface tension. Such an instability arises from classical continuum mechanics and is known as the Rayleigh instability [30]. The key to the stability of nanowires is quantum corrections to the classical stability analysis.

For wires aligned along the $z$-axis, the geometry is given by a function $R(z, \varphi)$ when using cylindrical coordinates. The linear stability of a nanowire with a given geometry can be analyzed by calculating the energy change $\delta \Omega$ caused by a small deformation $\delta R(z, \varphi)$ of the wire geometry. A stable realization of a nanowire requires an increase of $\Omega$ for all possible small deformations. A constraint on the deformation arises from the fact that, depending on material parameters, the deformed wire tries to find a compromise between a volume conserving deformation and one ensuring electroneutrality [28], leading to the constraint

$\mathscr{N} \equiv k_{\mathrm{F}}^{3} \mathscr{V}-\eta\left(3 \pi k_{\mathrm{F}}^{2} \mathscr{S} / 8\right)=$ const.

where $\mathscr{V}$ is the volume of the wire, $\mathscr{S}$ its surface area, and $k_{\mathrm{F}}=2 \pi / \lambda_{\mathrm{F}}$ the Fermi wavevector of the electrons. The parameter $\eta$ can be adjusted so as to fix the value of the effective surface tension to the material-specific value. In particular, $\eta=0$ corresponds to a constant-volume constraint, and $\eta=1$ is the constraint of constant Weyl charge. In this paper, the value $\eta=0.93$ is used, corresponding to the surface tension of $\mathrm{Na}(0.22 \mathrm{~N} / \mathrm{m}$ [31].)

The stability was first analyzed for cylindrical geometries and axisymmetric deformations, since these are the 
only destabilizing deformations in the classical calculation. In a semiclassical approach $[11,12]$, the grand canonical potential is divided into a smooth term depending on classical geometric quantities (volume, surface, mean curvature), referred to as the Weyl contribution [32], and a fluctuating quantum term. This quantum potential is calculated using a Gutzwiller-type trace formula [32,33]. One finds that for certain radii electronic shell effects suppress the classical Rayleigh instability at low temperatures. This leads to intervals of stable ('magic') radii, resulting in a series of stable cylindrical wires with conductance $\quad G / G_{0} \in\{1,3,5,6,8,10,12,14,17,23, \ldots\}$. Although all of those wires are predicted to be metastable, some of them $\left(G / G_{0}=5,14, \ldots\right)$ are expected to be barely stable, and thus unlikely to be observable. Since the semiclassical theory depends only trivially on the length of the wire, a puzzling conclusion is that nanowires with a magic radius remain stable whatever their length.

A full quantum calculation [13] shows that the suppression of the Rayleigh instability by electron shell effects is supplemented by an interplay between the Rayleigh and a novel Peierls-type instability missing in the semiclassical approximation. In fact, this latter quantum mechanical instability limits the maximal length of stable nanowires. For lengths of order $10-1000 \mathrm{~nm}$, the quantum calculation mainly confirms the set of magic conductance values mentioned above, but their corresponding intervals of stable radius values are reduced. Wires with a conductance of $5 G_{0}$ and $14 G_{0}$ are unstable and therefore missing.

Axial symmetry implies characteristic gaps in the sequence of stable nanowires, which is not fully consistent with the experimentally observed nearly perfect periodicity of the peak positions as a function of $\sqrt{G}$. The deviations can be accounted for neither by the inclusion of disorder [34], nor by the use of more elaborate self-consistent jellium models [35,36]. Since gaps in the sequence of cylindrical nanowires arise from a degeneracy of conductance channels, it is natural to assume that a Jahn-Teller deformation breaking the symmetry can lead to more stable deformed configurations.

In fact, the stability of nanowires with elliptic crosssections was recently examined theoretically [14] and stable elliptical nanowires were found. This extended analysis confirms the stability of the cylindrical wires found in the quantum calculation, even with respect to Jahn-Teller distortions. This includes the fact that cylindrical wires with a conductance of $5 G_{0}$ and $14 G_{0}$ are energetically unstable. Secondly, a number of stable elliptical nanowires with an aspect ratio $\varepsilon$ (which is defined as the ratio of the two major semiaxes of the ellipse) greater than one are found. The most stable elliptic wires have conductance values of $G / G_{0} \in$ $\{2,5,9, \ldots\}$.

Fig. 1 shows the corresponding stability diagram in the low conductance range as a function of temperature. Shaded areas indicate stability and the gray scale represents the value of the aspect ratio $\varepsilon$. The $x$-axis is given by the

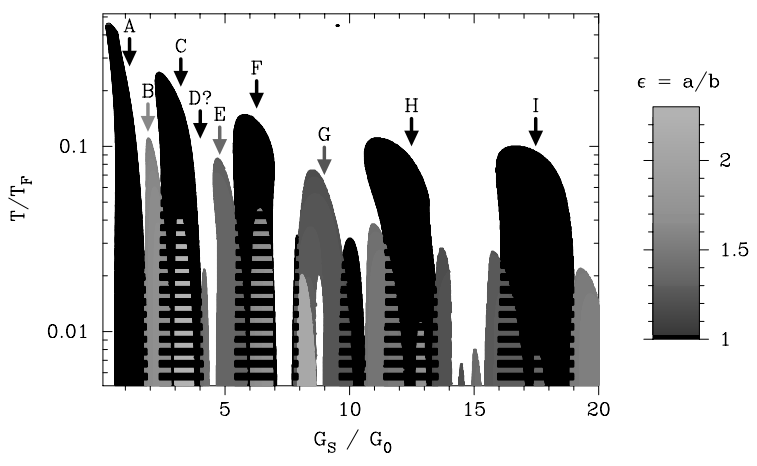

Fig. 1. Calculated energetically stable cylindrical and elliptical nanowires as a function of temperature (in units of the Fermi temperature $T_{\mathrm{F}}$ ). The surface tension is adjusted to a value of $0.22 \mathrm{~N} / \mathrm{m}(\eta=0.93)$, corresponding to $\mathrm{Na}$ [31]. The aspect ratio $\varepsilon$ is coded via the gray scale, and hatched areas indicate multistability.

corrected Sharvin conductance $G_{\mathrm{S}}$ of the wire [1]. The elliptical wire with conductance $9 G_{0}$ is expected to be much more stable at low temperature than the neighboring cylindrical wires at $8 G_{0}$ and $10 G_{0}$, because it is stable up to a much higher temperature.

Extending this analysis to higher conductance values and extracting the most stable configurations [14] leads to a sequence of stable cylindrical and elliptical nanowires that allows for a satisfying interpretation of the experiments on shell and supershell structure [16-18].

\section{Experiments}

Using remarkably simple experimental techniques, it is possible to gently break a metallic contact and thus form conducting nanowires. Besides the use of scanning tunneling microscopes (STM), the mechanically controllable break junction (MCBJ) is the most commonly used tool for the study of mechanical and transport properties of metal nanowires and point contacts. By indenting one electrode into another and then separating them, a stepwise decrease in electrical conductance is observed, down to the breakpoint when reaching a size of a single atom in cross section. In addition to the conductance, the STM and MCBJ techniques were used to study a variety of properties of metal nanowires, i.e. cohesive force [37], conductance fluctuations [38], thermopower [39], and shot noise [6]. The highly reactive alkali metals have been studied using a slightly modified MCBJ technique [4].

\subsection{Conductance histograms}

Nanowires fabricated by the methods discussed above show a wide variety of behaviors, with the atomic structure of the contacts playing an important role. For example, each scan of the dependence of conductance $G$ on the elongation $d$ is individual in detail as the atomic configuration of each 
contact may be widely different. Generally, the experiment does not allow for a detailed knowledge of the atomic-scale structure of the contacts. Nevertheless it is possible to extract information about possible quantization effects by performing a statistical analysis. Many individual conductance versus displacement curves together produce a histogram of the probability for observing a given conductance value, which is quite reproducible for a given metal and for fixed experimental parameters.

An important subject for study is the alkali metals, since they are nearly-free electron metals and therefore most closely approach the predictions of the nanoscale freeelectron model, described in the previous section. Fig. 2 shows conductance histograms for sodium recorded at different temperatures (taken from Ref. [16]). The curves are vertically offset for clarity and the amplitude has been normalized by the area under each graph. Each histogram is constructed from 1000-2000 individual scans. A number of peaks with very different intensity can be identified: Sharp peaks can be recognized at conductance values near 1, 3, 5 and $6 G_{0}$, while rather wide maxima are found near 9,12 and $17 G_{0}$. In addition, there is a small peak near $2 G_{0}$ and a very weak structure near $4 G_{0}$. Results similar to those for $\mathrm{Na}$ have been obtained for $\mathrm{Li}$ and $\mathrm{K}$.

\subsection{Further analysis of sodium histograms}

In order to obtain more information about the stable geometries that are realized in experiments, we have analyzed in detail the conductance histograms for sodium recorded in the temperature range of 20-70 K (Fig. 2). We fitted each data set with a function consisting of a sum of peaks plus a background. The conductance peaks were taken to be of Lorentzian shape with height, width and peak

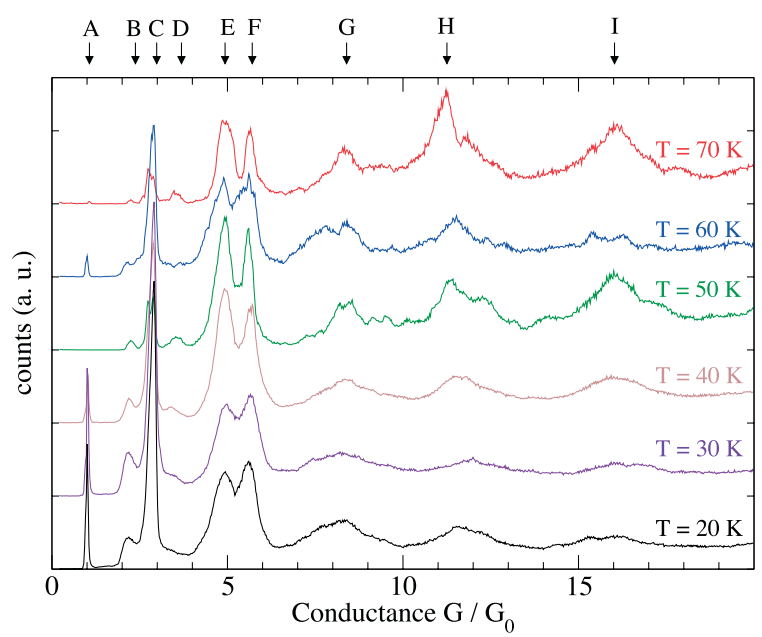

Fig. 2. Experimental conductance histograms for $\mathrm{Na}$ at different temperatures. The curves are vertically offset for clarity. The black arrows mark the approximate positions of the peaks that we consider in our analysis. (Data taken from Ref. [16]). position as fitting parameters. We have chosen to take nine peaks, corresponding to those that are clearly distinguishable in the data set. Their approximate positions are marked with black arrows in Fig. 2 and they are labeled with capital letters. This choice is not unique, as the fit can always be improved by including additional broad peaks of lower intensities. However, the parameters of additional peaks cannot be determined with high confidence, especially for noisy curves at higher temperatures. Fig. 3 shows the fit for the data at $T=30 \mathrm{~K}$ and the inset shows a fit of the region near $9 G_{0}$, including two additional peaks of lower intensity, for comparison.

The values of the fitting parameters now allow to analyze the development and the contributions of the different conductance peaks as a function of temperature. The peak positions stay almost constant with maximum fluctuations of three percent within the temperature range from 20 $70 \mathrm{~K}$. All the peaks have a finite (temperature dependent) width, and their positions are shifted downward compared to the theoretical quantized conductance values $G_{\mathrm{th}}$. As an exception, we find peak $B$ reproducibly at a higher conductance value than expected. This is possibly due to size constraints in building a nanowire of two channels by two atoms, which for a short wire will have a rather large tunnelling contribution of the third channel.

Table 1 lists average peak positions $\bar{G}_{\text {exp }}$, average peak widths $\overline{\Delta G}$, and theoretical conductance values $G_{\mathrm{th}}$ in columns 2-4. The last three columns sum up the results of the theoretical stability calculations, taking into account the most stable configurations only. The comparison with the experimental data shows good agreement in that one can ascribe a quantized conductance value to each experimental peak without ambiguity. In particular, it is possible to predict the shape of the nanowires seen in conductance histograms.

Peak D, with a conductance of $4 G_{0}$, is predicted to be

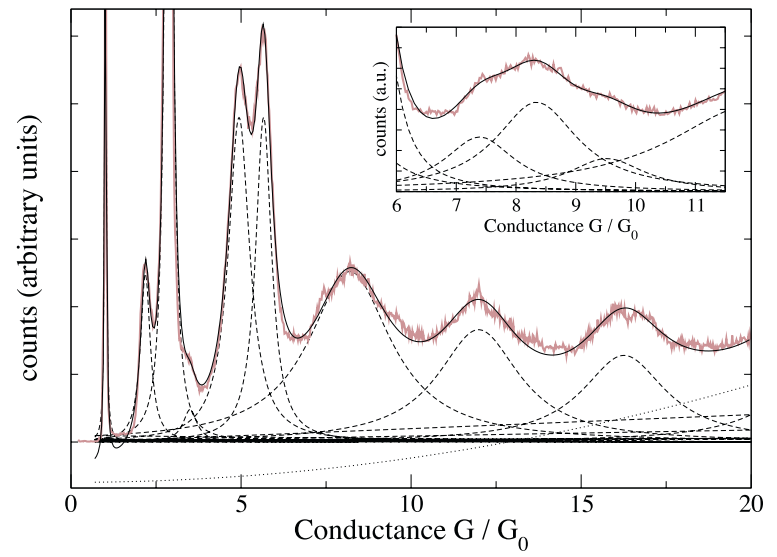

Fig. 3. Experimental conductance histogram data at $T=30 \mathrm{~K}$ fitted with Lorentzian peaks. The dashed lines show the individual peaks. Inset: A blow-up of the peak near $G=9 G_{0}$ fitted with two additional peaks of lower intensity is shown for comparison. 
Table 1

Experimental conductance, peak widths, theoretical conductance, and theoretical predicted shapes of the wires for the most important low conductance peaks

\begin{tabular}{llllll}
\hline Peak & $\bar{G}_{\text {exp. }}\left[G_{0}\right]$ & $\overline{\Delta G}\left[G_{0}\right]$ & $G_{\text {th }}\left[G_{0}\right]$ & Shape & $k_{\mathrm{B}} T_{\max }^{(\mathrm{Na})}(\mathrm{eV})$ \\
\hline A & 1.0 & 0.1 & 1 & Cylindrical & 1.50 \\
$\mathrm{~B}$ & 2.2 & 0.4 & 2 & Ellip. $\varepsilon=1.6$ & 0.36 \\
$\mathrm{C}$ & 2.8 & 0.2 & 3 & Cylindrical & 0.81 \\
$\mathrm{D}$ & 3.5 & 0.4 & 4 & 4-Fold? & $?$ \\
$\mathrm{E}$ & 4.9 & 0.6 & 5 & Ellip. $\varepsilon=1.3$ & 0.28 \\
$\mathrm{~F}$ & 5.6 & 0.5 & 6 & Cylindrical & 0.48 \\
$\mathrm{G}$ & 8.3 & 1.9 & 8 & Cylindrical & 0.11 \\
& & & 9 & Ellip. $\varepsilon=1.2$ & 0.24 \\
& & & 10 & Cylindrical & 0.10 \\
$\mathrm{H}$ & 11.6 & 2.0 & 12 & Cylindrical & 0.36 \\
$\mathrm{I}$ & 15.9 & 2.4 & 17 & Cylindrical & 0.32 \\
\hline
\end{tabular}

The right column lists the maximum temperature $T_{\max }$ up to which the wires are expected to remain stable, which is related to the depth of the corresponding energetic minimum. Peak $G$ is decomposed into three contributions as in the inset of Fig. 3.

barely stable in the present analysis. However, one might expect to find more stable nanowires with cross sections having a higher-order angular symmetry [40]. Such deformations cost significantly more surface energy, and can thus only be energetically favorable at low conductance. Therefore they are not likely to modify the stability diagram of Ref. [14] significantly, but one might in particular expect a wire with a four-fold angular symmetry to be stable at a conductance of $4 G_{0}$, which could explain the observation of peak D.

The relative weight (intensity) of each peak in the experimental histograms can also be extracted from the fit, and is shown in Fig. 4 at four different temperatures. The contribution of a stable wire to a histogram depends mostly on two conditions: (i) The wire has to be formed often enough to be statistically relevant; (ii) It needs to have a long enough lifetime so as to be recorded. The low-temperature

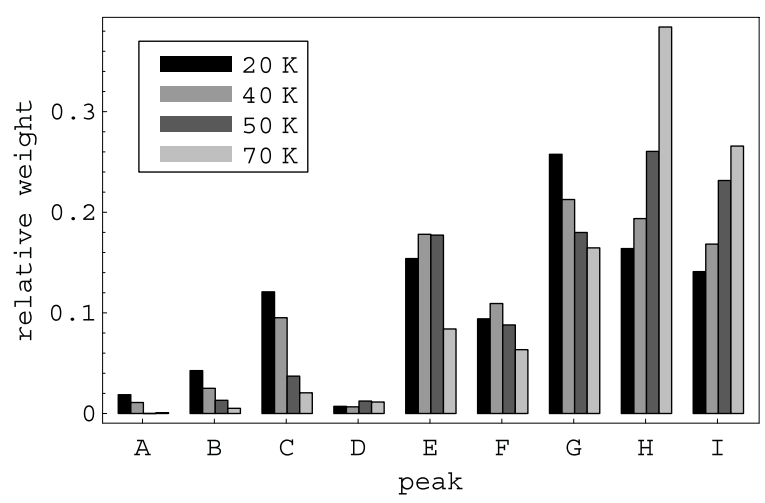

Fig. 4. Relative weights of the nine individual experimental conductance peaks (A to I) at four different temperatures, coded via the gray scale. lifetime of a nanowire is expected to increase with the maximum temperature up to which it is predicted to remain linearly stable. This is confirmed by recent calculations resticted to cylindrical nanowires [41]. Condition (i) depends on the ability of the system to probe new configurations, which is determined by the mobility of the atoms and the density of stable geometries in configuration space. Most of the very stable wires being cylindrical, one might expect that highly deformed wires have a low probability to form. This affects mainly the elliptic wire (B) with an aspect ratio of $\varepsilon=1.6$. The elliptical wires (E) and $(\mathrm{G})$ with a lower aspect ratio have a remarkably higher weight. Interestingly, peak (E) has a higher intensity than the neighboring cylindrical peak $(\mathrm{F})$. The big relative weight of peak $(\mathrm{G})$ is due to an overlay of contributions of an elliptical wire with $G=9 G_{0}$ and two cylindrical wires with $G=8 G_{0}$ and $G=10 G_{0}$ as suggested from the inset of Fig. 3. The decrease in the intensity of the low conductance peaks with growing temperature might be explained by thermally induced breaking of the contact.

\section{Conclusions}

We have presented a detailed analysis of experimental conductance histograms for Na nanowires. Comparison with a theoretical stability analysis of cylindrical and elliptical nanowires shows a one-to-one relation between observed peaks and theoretically stable geometries, and in particular allows a prediction of the cross-sectional shape of the wires.

\section{Acknowledgements}

This research has been supported by the DFG through SFB 276 and the EU Network DIENOW. JB and CAS acknowledge support from NSF grant DMR0312028.

\section{References}

[1] N. Agrait, A. Levy Yeyati, J.M. van Ruitenbeek, Phys. Rep. 377 (2003) 81.

[2] N. Agraï, J.G. Rodrigo, S. Vieira, Phys. Rev. B 47 (1993) 12345.

[3] M. Brandbyge, J. Schiøtz, M.R. Sørensen, P. Stoltze, K.W. Jacobsen, J.K. Nørskov, L. Olesen, E. Lægsgaard, I. Stensgaard, F. Besenbacher, Phys. Rev. B 52 (1995) 8499.

[4] J.M. Krans, J.M. van Ruitenbeek, V.V. Fisun, I.K. Yanson, L.J. de Jongh, Nature 375 (1995) 767.

[5] E. Scheer, N. Agraït, J.C. Cuevas, A. Levy Yeyati, B. Ludoph, A. Martín-Rodero, G. Rubio Bollinger, J.M. van Ruitenbeek, C. Urbina, Nature 394 (1998) 154.

[6] H.E. van den Brom, J.M. van Ruitenbeek, Phys. Rev. Lett. 82 (1999) 1526.

[7] Y. Kondo, K. Takayanagi, Phys. Rev. Lett. 79 (1997) 3455. 
[8] C.A. Stafford, D. Baeriswyl, J. Bürki, Phys. Rev. Lett. 79 (1997) 2863.

[9] J. van Ruitenbeek, M. Devoret, D. Esteve, C. Urbina, Phys. Rev. B 56 (1997) 12566.

[10] C. Yannouleas, E. Bogachek, U. Landman, Phys. Rev. B 57 (1998) 4872.

[11] F. Kassubek, C.A. Stafford, H. Grabert, R.E. Goldstein, Nonlinearity 14 (2001) 167.

[12] C.-H. Zhang, F. Kassubek, C.A. Stafford, Phys. Rev. B 68 (2003) 165414.

[13] D.F. Urban, H. Grabert, Phys. Rev. Lett. 91 (2003) 256803.

[14] D.F. Urban, J. Bürki, C.H. Zhang, C.A. Stafford, H. Grabert, cond-mat/0312517, 2003.

[15] W.A. Heer, Rev. Mod. Phys. 65 (1993) 611.

[16] A.I. Yanson, I.K. Yanson, J.M. van Ruitenbeek, Nature 400 (1999) 144.

[17] A.I. Yanson, I.K. Yanson, J.M. van Ruitenbeek, Phys. Rev. Lett. 84 (2000) 5832.

[18] A.I. Yanson, I.K. Yanson, J.M. van Ruitenbeek, Fizika Nizkikh Temperatur 27 (2001) 1092.

[19] U. Landman, W.D. Luedtke, N.A. Burnham, R.J. Colton, Science 248 (1990) 454.

[20] T.N. Todorov, A.P. Sutton, Phys. Rev. B 54 (1996) R14234.

[21] M.R. Sørensen, M. Brandbyge, K.W. Jacobsen, Phys. Rev. B 57 (1998) 3283.

[22] M. Brack, Rev. Mod. Phys. 65 (1993) 677.

[23] S. Datta, Electronic Transport in Mesoscopic Systems, Cambridge University Press, Cambridge, 1995.

[24] J.A. Torres, J.I. Pascual, J.J. Sáenz, Phys. Rev. B 49 (1993) 16581 .

[25] J. Bürki, C.A. Stafford, X. Zotos, D. Baeriswyl, Phys. Rev. B 60 (1999) 5000.
[26] F. Kassubek, C.A. Stafford, H. Grabert, Phys. Rev. B 59 (1999) 7560.

[27] J. Bürki, C.A. Stafford, Phys. Rev. Lett. 83 (1999) 3342.

[28] C.A. Stafford, F. Kassubek, J. Bürki, H. Grabert, Phys. Rev. Lett. 83 (1999) 4836.

[29] C.A. Stafford, F. Kassubek, J. Bürki, H. Grabert, D. Baeriswyl, in: D.C. Glattli, M. Sanquer, J. Tran Thanh (Eds.), Quantum Physics at the Mesoscopic Scale, EDP Sciences, Les Ulis, France, 2000, pp. 49-53.

[30] S. Chandrasekhar, Hydrodynamic and Hydromagnetic Stability, Dover, New York, 1981

[31] W.R. Tyson, W.A. Miller, Surf. Sci. 62 (1977) 267.

[32] M. Brack, R.K. Bhaduri, Semiclassical Physics, Frontiers in Physics, 96, Addison Wesley, Reading, MA, 1997.

[33] M.C. Gutzwiller, Chaos in Classical and Quantum Mechanicanics, Addison Wesley, Reading, MA, 1997.

[34] J. Bürki, C.A. Stafford, in: T. Martin, G. Montambaux, J. Tran Thanh Van (Eds.), Electronic Correlations: from Meso- to Nano-Physics, EDP Sciences, Les Ulis, France, 2001, pp. 27-30.

[35] M.J. Puska, E. Ogando, N. Zabala, Phys. Rev. B 64 (2001) 033401.

[36] E. Ogando, N. Zabala, M.J. Puska, Nanotechnology 13 (2002) 363.

[37] G. Rubio, N. Agraï, S. Vieira, Phys. Rev. Lett. 76 (1996) 2302 .

[38] B. Ludoph, J.M. van Ruitenbeek, Phys. Rev. B 61 (2000) 2273.

[39] B. Ludoph, J.M. van Ruitenbeek, Phys. Rev. B 59 (1999) 12290 .

[40] J. Bürki, D.F. Urban, C.A. Stafford, in preparation.

[41] J. Bürki, C.A. Stafford, D.L. Stein, Cond-mat/0406374/. 